\title{
A Internet das coisas como recurso interativo na aprendizagem: Benefícios percebidos por discentes de Ciências Contábeis*
}

\author{
Internet de las cosas como un recurso interactivo en el aprendizaje: Beneficios percibidos por los \\ Estudiantes de Contabilidad \\ The Internet of things as an interactive learning resource: Benefits perceived by Accounting Students
}

Alcir Junior Garcia da Matta

Universidade Federal do Rio Grande, Brasil

DOI: https://doi.org/10.11144/Javeriana.cc22.icri

ORCID: https://orcid.org/0000-0001-5842-8286

Carla Milena Gonçalves Fernandes

Aprovação: 20/08/2020

Universidade Federal do Rio Grande, Brasil

Publicação: 18/08/2021

ORCID: https://orcid.org/0000-0002-9646-4592

Anderson Betti Frare

Universidade Federal de Santa Catarina, Brasil

ORCID: https://orcid.org/0000-0002-4602-7394

Alexandre Costa Quintana ${ }^{\mathrm{a}}$

Universidade Federal do Rio Grande, Brasil

professorquintana@hotmail.com

ORCID: https://orcid.org/0000-0001-6896-9465

\section{Resumo:}

A tecnologia, principalmente no âmbito contábil, está mudando a forma como produzimos, trabalhamos, estudamos e nos relacionamos. Este estudo tem como objetivo analisar o efeito da preocupação com a Internet das Coisas na aprendizagem e nos benefícios percebidos por estudantes do curso de Ciências Contábeis em uma Universidade Pública Federal. Os dados foram coletados por meio de surveys e analisados com Modelagem de Equações Estruturais. Os resultados apontam que o envolvimento dos estudantes é um fator chave para a preocupação com a Internet das Coisas na aprendizagem, e isto influencia os benefícios percebidos por eles. Ademais, o número de serviços de Internet das Coisas e a compatibilidade percebida também influenciam significativamente os benefícios percebidos pelos estudantes. $\mathrm{O}$ estudo contribui teoricamente ao enumerar os antecedentes dos benefícios percebidos pelos docentes e, principalmente, pelos discentes.

Códigos JEL: L63, L89.

Palavras-chave: Internet das coisas, recursos interativos na aprendizagem, discentes de contabilidade, educação contábil.

\section{Resumen:}

La tecnología, especialmente en el campo de la contabilidad, está cambiando la forma en que producimos, trabajamos, estudiamos y nos relacionamos. El objetivo de este estudio es analizar el efecto de la preocupación con el Internet de las Cosas en el aprendizaje y en los beneficios percibidos por los estudiantes del curso de Ciencias Contables en una Universidad Pública Federal. Los datos fueron recolectados por encuesta y analizados utilizando el modelo de ecuaciones estructurales. Los resultados muestran que la participación de los estudiantes es un factor clave para la preocupación con el Internet de las cosas en el aprendizaje, y esto influye en los beneficios percibidos por estos. Además, la cantidad de servicios de Internet de las cosas y la compatibilidad percibida también influyen significativamente en los beneficios percibidos por los estudiantes. El estudio contribuye teóricamente al enumerar los antecedentes de los beneficios percibidos por los maestros y, principalmente, por los estudiantes.

Códigos JEL: L63, L89.

Palabras clave: Internet de las cosas, recursos de aprendizaje interactivo, estudiantes de contabilidad, educación contable.

Autor notes a Autor de correspondência. E-mail: professorquintana@hotmail.com 


\begin{abstract}
:
Technology, especially in the accounting field, is changing the way we produce, work, study and relate. This study aims to analyze the effect of the concern with the Internet of things on learning and the benefits perceived by students of the Accounting Sciences course at a Federal Public University. Data were collected by survey and analyzed using Structural Equation Modeling. The results show that student involvement is a key factor for the concern with the Internet of things in learning, and this influences the benefits perceived by these students. Besides, the number of Internet of things services and the perceived compatibility significantly influence the benefits perceived by students. The study contributes theoretically by listing antecedents of the perceived benefits by teachers and especially by students.
\end{abstract}

JEL Codes: L63, L89.

Keywords: Internet of things, interactive learning resources, accounting students, accounting education.

\title{
Introdução
}

A tecnologia avança numa velocidade difícil de ser acompanhada e com isso a forma de comunicar-se muda, fazendo com que as pessoas tenham que adaptar-se a uma nova era digital. Dentre os mecanismos tecnológicos em destaque cita-se a Internet das Coisas (IoT por suas siglas em inglês). A IoT encontra-se intrinsecamente relacionada com a Tecnologia da Informação (TI) e consiste em expandir e intensificar por meio de redes, sensores e inteligência artificial as relações entre as pessoas e objetos (coisas) inteligentes, servindo assim para além de conectar pessoas, coletar, automatizar, gerenciar e analisar informações (Abdel-Basset, Manogaran, Mohamed \& Rushdy, 2018), insere-se a exemplo casas e cidades inteligentes (Wu, Xiong, \& Li, 2019).

Ao vincular a IoT como um elemento que otimize o processo de ensino e aprendizagem, em especial na área das ciências contábeis denota-se o vínculo existente desta com a área de TI, tendo como uma das prerrogativas básicas da junção que os futuros contadores sejam proativos e busquem adequarem-se as mudanças tanto no que diz respeito a contabilidade como a tecnologia (Hatane, Johari, Valencia \& Prayug, 2019). Para Ghani e Muhammad (2019), tal mudança deve ser iniciada na universidade. Nesse sentido, os futuros profissionais poderão tornar-se capazes de desenvolverem habilidades criativas, sociais e tecnológicas frente às distintas e dinâmicas informações disponibilizadas a todo o momento.

A IoT surge como uma nova possibilidade de conexão entre professor-aluno e vice-versa, bem como um aporte facilitador para as atividades curriculares (Abdel-Basset et al., 2018), ou seja, pode ser útil ao servir como um dos recursos educacionais a serem introduzidos ao ensino contábil. "Os profissionais de contabilidade veem a necessidade de que os graduandos em contabilidade tenham foco na aprendizagem e educação contínuas, particularmente na aquisição de conhecimento e habilidades em TI" (Ghani \& Muhammad, 2019, p. 19).

Abdel-Basset et al. (2018) mencionam que "as salas de aula devem ter quadros e displays digitais (...) gravadores de vídeo de alto desempenho para captura de palestras e que devem estar disponíveis na escola" (Abdel-Basset et al., 2018, p. 11). Porém, um dos principais entraves é a falta de acessibilidade por parte de alguns estudantes as ferramentas tecnológicas.

Abdel-Basset et al. (2018), Ghani e Muhammad (2019), Hatane et al. (2019) e Wu et al. (2019) os quais expõem acerca da preocupação existente com a aplicação de recursos interativos na aprendizagem e que a efetivação da IoT no campo educacional pode agir como elemento potencializador de que possíveis benefícios possam ser percebidos pelos estudantes da área contábil. Alguns dos aspectos pertencentes à inserção de recursos interativos e que tem como premissa facilitar a aprendizagem envolvem a acessibilidade (Abdel-Basset et al., 2018; Conforto \& Santarosa, 2002), o envolvimento (Lim, 2017; Schwab \& Freitas, 2016) e o tempo (Abdel-Basset et al., 2018; Ghani \& Muhammad, 2019; Hsu \& Lin, 2016; Watty, Mckay \& Ngo, 2014).

Em relação com a IoT e os recursos interativos na aprendizagem encontram-se os chamados benefícios percebidos e, dentro destes, as externalidades de rede direta e indireta. Estas externalidades envolvem 
basicamente a percepção das pessoas quanto a alguns atributos como: o número de serviços de IoT disponíveis, a massa crítica percebida, a compatibilidade e a complementaridade. As duas primeiras, relacionadas à rede direta, são atributos que buscam especificar a disponibilidade existente de serviços que envolvam a IoT e a aceitação das pessoas diante do avanço tecnológico. Já os dois últimos atributos, relacionados à externalidade de rede indireta, de forma geral, dizem respeito a quanto mais pessoas utilizarem determinados dispositivos tecnológicos (aplicativos, softwares, compartilhamento em rede) com o intuito de obterem produtos, serviços, informações, etc., este IoT representará maior valor a partir do momento em que o número de usuários aumentar (Hsu \& Lin, 2016).

Com o advento do uso das tecnologias na educação e particularmente no campo contábil infere-se a relevância que os recursos tecnológicos têm a oferecer para dinâmica entre discentes e/ou docentes tanto em sala de aula como fora dela (Abdel-Basset et al., 2018). No entanto, estudos que demonstraram o vínculo existente e positivo entre acadêmicos de contabilidade e não somente a aceitação da IoT (Hatane et al., 2019), mas também o uso efetivo de ferramentas oriundas da IoT no ambiente acadêmico e sua implicação empírica em estudantes de contabilidade inferem a precariedade da aplicação da referida tecnologia no processo de ensino e aprendizagem.

Igualmente, Ghani e Muhammad (2019), Hatane et al. (2019) e Wu et al. (2019) afirmam que o processo para o desenvolvimento e ampliação da IoT é ainda incipiente, especialmente na área contábil. Neste sentido, Hsu \& Lin (2016) assinalam a falta de estudos empíricos que possam fortalecer ainda mais o vínculo entre a contabilidade (discentes, docentes/profissionais) com os sistemas informacionais.

Este estudo tem como objetivo analisar o efeito da preocupação com a Internet das Coisas na aprendizagem com os benefícios percebidos por estudantes do curso de Ciências Contábeis em uma Universidade Pública Federal do Brasil.

Além das ponderações mencionadas justifica-se o estudo em virtude do uso da IoT e de seus dispositivos tecnológicos servirem como aliados no processo educacional (Abdel-Basset et al., 2018). O uso da tecnologia IoT está promovendo uma gradativa disrupção no processo de ensino e aprendizagem (Cornel, 2015).

Tão logo, para Abdel-Basset et al. (2018) existem alguns pontos contributivos para a inserção da IoT no âmbito educacional: i) "criar um clima inovador para os alunos"; ii) "minimiza o tempo gasto frente aos métodos tradicionais de ensino"; iii) há a possibilidade de "melhorar o gerenciamento do tempo"; iv) almejar "valência para todos". Destarte, os dispositivos, ferramentas e aplicativos disponíveis podem "transforma alunos em criadores: o grande número de dispositivos vinculados e a tomada de decisão é a melhor oportunidade para os alunos entenderem, criarem e se controlarem” (Abdel-Basset et al., 2018, p. 11).

Não obstante, investigar a aceitação da tecnologia IoT e seu uso por estudantes de Contabilidade também se configuram de maneira emergente, visto os benefícios que o uso da tecnologia pode proporcionar aos discentes (Hatane et al., 2019). Este estudo pode ter implicações teóricas, acerca da preocupação com os recursos interativos e a possível influência nos benefícios percebidos do uso da IoT na aprendizagem, por parte dos estudantes de Ciências Contábeis. De forma prática, existe a implicação de possíveis remodelagens no ensino, bem como de um novo olhar frente à demanda provocada por essa tecnologia disruptiva.

\section{Referencial conceitual e desenvolvimento das hipóteses}

\section{Internet das Coisas (IoT)}

A tecnologia pode resumir-se em um processo imaginativo no qual a sociedade pode utilizar-se de recursos materiais e imateriais, ou criá-los a partir do que está disponível na natureza e nas suas circunstâncias possíveis, com a finalidade de encontrar respostas para os problemas de sua composição. É indiscutível a importância 
da tecnologia para as pessoas em vários aspectos, pois com ela, ganhamos tempo e eficiência em diversas atividades rotineiras ( $\mathrm{Lin} \& \mathrm{Lu}, 2011)$.

Neste processo de mudança, a Internet das Coisas surge para facilitar várias atividades, como agilidade "no processo de coleta e processamento de dados em informações úteis para a tomada de decisões” (Hatane et al., 2019, p. 273). Por conseguinte, a IoT representa uma visão em que a internet se estende para o mundo real envolvendo objetos do cotidiano. Os itens físicos não são mais desconectados do mundo virtual, mas podem ser controlados remotamente e podem atuar como pontos de acesso físico aos serviços de internet. Além disso, a IoT torna a computação verdadeiramente onipresente. A origem do nome Internet das Coisas é atribuída a Kevin Ashton que a definiu como uma ideia de embutir sensores em objetos do dia a dia (AbdelBasset et al., 2018).

De forma geral, a IoT pode se definir como a arte que protagoniza a interação entre o mundo físico e o digital, na qual os dispositivos e objetos com a capacidade de interceptar, analisar e processar dados que estão conectados de alguma forma à internet, podem se comunicar em tempo real e reagir de forma autêntica, de acordo com certos questionamentos realizados (Abdel-Basset et al., 2018).

No que tange ao uso de tecnologias sob o viés acadêmico, por exemplo, em sala de aula, cada vez mais estão sendo criados e aperfeiçoados dispositivos e recursos para aproximar a educação da realidade dos alunos (Silva \& Szesz Junior, 2018). Neste processo de transição os sistemas informacionais ou mesmo a tecnologia 4.0 (exemplo IoT), que atinge a área contábil, exige assim profissionais que atentem para as exigências do mercado (Ghani \& Muhammad, 2019).

Com a aplicação da tecnologia na educação nota-se como característica general, a utilização de uma grande diversidade de recursos tecnológicos para fins pedagógicos, com a finalidade de facilitar o aprendizado. Por médio de esta podem se obter mais conhecimentos e uma capacidade de rendimento maior (Tachizawa \& Andrade, 2003). Outrossim, essa situação precisa, segundo os autores, ser absorvida pelas universidades para formar novos profissionais que saibam lidar com esta nova realidade. Assim, o processo pelo qual um indivíduo possa obter conhecimento de forma mais simples e completa, passa pela busca, acesso, seleção e aplicação das informações (Lin \& Lu, 2011).

Uma das conjecturas que se pode traçar ao relacionar a IoT aplicada à educação tange à tentativa das instituições de ensino de se adaptar frente à inserção de novos dispositivos no campo educacional. Além disso, este processo de adaptação implica para os estudantes um maior engajamento quando estes forem entrar no mercado de trabalho (Cornel, 2015).

Para Wu et al. (2019) “a IoT é uma instalação de rede dinâmica e global com recursos de autoconfiguração baseados em padrões e protocolos de comunicação interoperáveis” (p. 100090). Assim, a existência de canais de comunicação, apoiados na tecnologia presente nos mais variados meios, e que conectam os atores educacionais (estudantes, professores, instituição), torna-se de uma relevância significativa para o desenvolvimento do ensino e aprendizagem. Para Hatane et al. (2019) ao inserir a IoT no âmbito educacional, e mais especificamente na área das Ciências Contábeis, têm-se a capacidade de instigar docentes e discentes à busca pelo aprimoramento de informações, além de server como um estímulo para o desenvolvimento tanto de habilidades criativas como sociais. Igualmente, este processo estimula aos estudantes a descobrir novas habilidades que os possibilite tornarem-se profissionais adaptáveis aos mecanismos tecnológicos.

Hatane et al. (2019) e Ghani e Muhammad (2019) realizaram os primeiros estudos empíricos aplicados a estudantes, avaliando o uso da IoT na área das ciências sociais aplicadas e particularmente no campo da contabilidade. Hatane et al. (2019) objetivaram verificar a aceitação do uso da IoT por parte de discentes em contabilidade de universidades públicas e privadas da Indonésia. A partir do retorno de 306 respondentes puderam constatar, por meio da técnica quantitativa de análise fatorial, que o nível de confiança para com o uso da tecnologia é similar entre homens e mulheres.

Estes estudos ressaltam que as habilidades (criativas, sociais e tecnológicas) não são determinadas pelo nível de inteligência. De forma geral, todos os estudantes são capazes de impulsionar suas próprias habilidades para 
compreender a IoT, de maneira que esta possa auxiliá-los a otimizar seu tempo, trabalho, bem como suas necessidades profissionais (Hatane et al., 2019).

Já o estudo de Ghani e Muhammad (2019) buscou "examinar as expectativas dos empregadores quanto ao conhecimento e habilidades em graduandos de contabilidade antes de contratá-los” (p. 19). Tais conhecimentos e habilidades sob a ótica da indústria 4.0 convergem para a temática IoT. Os autores realizam uma pesquisa qualitativa por meio de entrevistas tendo como participantes 1 contador, 1 auditor e 2 acadêmicos de contabilidade de uma universidade pública localizada na Malásia.

Os resultados apontaram que os profissionais percebem que a tecnologia vem tomando espaço em todas as atividades humanas. Esta percepção é também compartilhada pelos acadêmicos, o que abre espaço à ideia de que os estudantes precisam prepararem-se para essas mudanças no mercado do trabalho. Nesse sentido, há uma necessidade de que as tecnologias possam ser instrumentalizadas no ambiente acadêmico para que dessa forma haja um aprimoramento dos futuros contadores frente as necessidades do mercado.

\section{Recursos interativos na aprendizagem}

Com o avanço das tecnologias e o alcance da internet, informações são pesquisadas na rede para a realização das mais rotineiras atividades. Quando surgem dúvidas sobre determinados assuntos ou é preciso resolver algum problema, lá está o Google para trazer as respostas que precisamos (Watty et al., 2014). Assim, ao pesquisar estamos buscando subsídios que possam fomentar as mais diversas necessidades sejam elas pessoais e/ou profissionais. Nesse sentido, ao relacionar os avanços tecnológicos com os recursos interativos que viabilizem a aprendizagem pode-se mencionar que, segundo Watty et al. (2014) estes recursos referem-se a acessibilidade, envolvimento e tempo.

Para Conforto e Santarosa (2002) ter acesso a tecnologia é quebrar barreiras nos ambientes virtuais. Tornar uma página acessível é permitir que diferentes tipos de pessoas, com deficiência ou não, possam chegar aos conteúdos e compreendê-los com autonomia. A democratizando da acessibilidade à tecnologia permite, principalmente nas universidades, desenvolver as habilidades dos discentes.

É, por tanto, necessária uma atenção especial à acessibilidade quando se pretende aplicar algum recurso tecnológico a estudantes, de modo que todos possam realmente ver-se beneficiados. Abdel-Basset et al. (2018) ainda arguem que, intrínseca à educação, a IoT pode servir para a inserção de "aplicativos educacionais, livros eletrônicos, sistemas de rastreamento de presenças" etc. (p. 9).

A pesquisa realizada por Hatane et al. (2019) verificou que $86 \%$ dos respondentes buscavam acesso a internet por smartphones em vez do computador. Dai que o acesso a dispositivos móveis "tem o potencial de não apenas afetar como vivemos, mas também como podemos trabalhar de forma prático e útil” (p. 273).

A acessibilidade compreende um elemento importante quando se trata da interação de estudantes e dos meios tecnológicos. No entanto, em países que possuem um desenvolvimento crescente em dispositivos tecnológicos, esse fator (acessibilidade) não apresenta evidências consolidadas do seu papel na preocupação com recursos interativos na aprendizagem (Kanwal \& Rehman, 2017). Nesse aspecto, formulou-se a seguinte hipótese:

$\mathbf{H}_{1 \mathrm{~A}}$ : A Acessibilidade influencia positivamente a preocupação com recursos interativos da aprendizagem.

A forma como nos envolvemos com esses novos recursos gera, na maioria das vezes, benefícios. Com a internet, por exemplo, temos acesso a um volume de informações incalculáveis e de forma quase instantânea. Com isso, esse envolvimento com a rede nos expõe a uma enorme gama de benefícios na aprendizagem, como por exemplo, o fortalecimento de um engajamento entre estudantes (Schwab \& Freitas, 2016).

Para Warren, Sulaiman e Jaafar (2014) o envolvimento é uma das formas que mais estimula a troca de ideias. Isto acontece porque as pessoas tornam-se mais engajadas a partir do momento em que percebem que outros também se mostram interessados em determinados assuntos, como por exemplo, a imersão em um novo aparato tecnológico. Já Astin (1984) mostra a importância de analisar o desenvolvimento de estudantes 
por meio de seu envolvimento diante de experiências acadêmicas que possam propiciar sua participação ativa, ou seja, instigar uma relação diante dos recursos interativos promovidos pelos docentes.

Adiciona-se que para que o critério envolvimento seja favorável à ocorrência do recurso interativo o "professor precisa ter uma variedade de habilidades e conhecimentos em TI, a fim de adaptar e usar a TI para (ajudar ao estudante) no processo de ensino e aprendizagem”. (Ghani \& Muhammad, 2019, p. 24). Isto principalmente quando se busca concatenar o envolvimento aos meios tecnológicos em prol da aprendizagem (Heiberger \& Harper, 2008, p. 22).

Kanwal e Rehman (2017) mencionam que dependendo do tipo de ferramenta tecnológica a ser inserida no processo de ensino e aprendizagem, se faz necessário que esta forneça "funções de entretenimento interativo junto com salas de bate-papo e painéis de mensagens". "Isso pode levar ao envolvimento do usuário." (p. 10971). Além disso, Northey, Bucic, Chylinski et al. (2015) expõem que

o envolvimento dos alunos é uma preocupação constante dos educadores, devido à sua associação positiva com a aprendizagem. (Para tanto) a abordagem de aprendizagem centrado no aluno é essencial nessa transição, porque propicia engajamento dos alunos, situando-os como parceiros instrumentais no processo de aprendizado. (Northey et al., 2015, p. 1)

Assim, elabora-se a seguinte hipótese:

$\mathrm{H}_{1 \mathrm{~B}}$ : O Envolvimento influencia positivamente a preocupação com recursos interativos da aprendizagem.

Apesar de vivermos em uma era digital, nem todos possuem tempo necessário para desenvolver ou aprender novas tecnologias, bem como conseguir ampliar os seus conhecimentos e habilidades com as novidades que surgem (Watty et al., 2014). Peleias, Guimarães, Chan e Carlotto (2018) acrescentam que estudantes costumam ter "dupla jornada o que pode levar o trabalho a concorrer com a vida acadêmica" (p. 31). Assim, os autores aduzem que "estudantes brasileiros de contabilidade normalmente trabalham de dia e estudam a noite" caracterizando assim a dupla jornada (Peleias et al., 2018, p. 32).

O gerenciamento do tempo, principalmente para estudantes universitários, é um fator a ser observado, pois estes necessitam de orientações que possam fazer com que desenvolvam suas habilidades, bem como conseguir otimizar seu tempo. Isto pode refletir na aprendizagem ao passo que exista a adoção por parte dos docentes de introduzir o uso de aparatos tecnológicos em suas aulas (David, Helou \& Rahim, 2012).

Argumenta-se, ainda, que o uso de tecnologia baseada na IoT no processo de ensino e aprendizagem diz respeito aos

ambientes híbridos de aprendizado que são criados através da fusão do tempo formal de aprendizado com atividades informais fora da sala de aula. (No presente século) os materiais para o processo de aprendizado tornaram-se centralizados e personalizados, removendo as fronteiras entre o aprendizado, vida profissional e privada. (Abdel-Basset et al., 2018, p. 9).

Ghani e Muhammad (2019) afirmam que em um horizonte temporal expressado por meio da evolução tecnológica, o tempo é um dos principais elementos para que os futuros contadores e consequentemente as práticas contábeis ganhem "precisão e pontualidade na melhoria da eficiência e garantia de dados, além de outros fins de tomada de decisão... (Como consequência) os contadores precisam ser versados nas novas tecnologias para permanecerem" (Ghani e Muhammad, 2019, p. 20) firmes e atuantes ao entrarem no mercado de trabalho.

Por tanto, a maximização do tempo, como um fator de pertinência relacionado a aprendizagem a partir do uso de tecnologia, surge no sentido de que quanto mais os usuários perceberem que tais mecanismos estão satisfazendo suas necessidades, maior será, segundo os autores, o envolvimento destes não somente em nível pragmático como de sociabilidade (Warren, Sulaiman, \& Jaafar, 2014).

Nesse sentido, define-se a seguinte hipótese:

$\mathbf{H}_{1 \mathrm{C}}$ : $\mathrm{O}$ Tempo influencia positivamente a preocupação com recursos interativos da aprendizagem. 


\section{Benefícios percebidos}

Como potenciais influenciadores dos benefícios percebidos, encontram-se as externalidades de rede que são conceitos usados para compreender as evoluções da sociedade, assim certas ideias que foram sendo induzidas a determinados grupos e que foram aumentando o número de indivíduos que a elas foram gradualmente inclusos.

À medida que os serviços de redes sociais, como o twitter aumentam sua popularidade eles acabam por tornarem-se cada vez mais importantes para os usuários. Um dos principais fatores a se levar em conta na hora de escolher entre duas redes sociais seja o facebook ou twitter é procurar saber em qual está à maior parte de seus amigos (Chiu, Ramirez \& Ruiz, 2013).

Nesse contexto, as externalidades de rede podem ser divididas entre diretas e indiretas. Para exemplificar, nas externalidades de rede direta, aqui se faz a seguinte análise com base em dois tipos, números de serviços de IoT e massa crítica percebida de usuários (Gupt \& Mela, 2008).

Quanto ao número de serviços de IoT, ele acaba por definir o lado da oferta da rede, já que o número de pessoas acaba por definir o lado da demanda (Hsu \& Lin, 2016). Com o aumento da demanda, o número crescente fez com que novos pontos de serviços e recursos de IoT sejam adicionados em uma variedade de locais. Por exemplo, ela pode ser encontrada em fechaduras inteligentes, drones, carros automáticos, aplicativos para controlar o sono, caminhadas/corridas, venda/compras de produtos, entre outros (Katz \& Shapiro, 1985).

Para Hatane et al. (2019) a combinação dos termos inovação e serviços criativos podem fornecer aos profissionais contábeis habilidades criativas, sociais, móveis e de navegação na busca de informações. No entanto, se tais habilidades forem operacionalizadas na academia as chances dos futuros profissionais alocarem-se, não somente no mercado de trabalho, mas nas atividades que desejam, tornam-se possíveis.

Outrossim, a evolução tecnológica propicia mais possibilidades de conectividade "e ambientes inteligentes para melhorar a qualidade de vida e a produtividade no trabalho cita-se por exemplo, dispositivos de economia como o termostato Nest que são uma extensão do smartphone, chamadas de serviços automáticos, informações de trânsito em tempo real, etc." (Hatane et al., 2019, p. 517).

Northey et al. (2015) acrescentam que ao

fornecer aos alunos recursos, como conteúdo, ideias, tarefas de aprendizagem e apoiá-los através de sistemas ativados por ferramentas interativas, os educadores concedem oportunidades de interações assíncronas para a apoiar a aprendizagem relacionada ao curso. (No uso de) métodos de entrega de serviços educacionais, (os docentes podem) utilizar efetivamente as ferramentas disponíveis para envolver os alunos. (Northey et al., 2015, pp. 4-7)

Por tanto, cria-se a seguinte hipótese:

$\mathbf{H}_{2 \mathrm{~A}}$ : O Número de serviços de IoT influencia positivamente os benefícios percebidos pelos estudantes de contabilidade.

Em relação à massa crítica de usuários, quando um determinado aplicativo começa a ganhar cada vez mais notoriedade seu valor aumenta. Sendo assim, surgem avaliações, sejam elas positivas ou negativas em que sua popularidade aumenta porque há uma massa crítica de usuários desse modelo (Lou, Luo \& Strong, 2000).

Além disso, Hsu e Lin (2016) arguem que a massa crítica percebida compreende que quanto mais pessoas utilizarem determinados recursos e/ou dispositivos tecnológicos maior será o aceite dos usuários. Isto faz com que estes percebam a possibilidade de benefícios com o uso de ferramentas tecnológicas. Lin e Bhattacherjee (2008), por sua parte, afirmam que o termo massa crítica denota uma interatividade do número de usuários adotantes de tecnologias de informação.

No meio acadêmico, quanto maior o nível de interação do estudante com a IoT, mais favorável será a conectividade entre os estudantes. Por tanto, ao parecer existe uma relação entre o nível de interatividade e a integração social. Tão logo possam os benefícios ser percebidos com maior intensidade para fins acadêmicos 
do que para entretenimento, percebe-se que "a eficácia educacional relaciona-se à capacidade de aumentar a participação" dos estudantes, e logo, a massa crítica pode influenciar os benefícios percebidos (Heibergert \& Harper, 2008, p. 24).

Tem-se assim a hipótese $\mathbf{H}_{2 \mathrm{~B}}$ : A massa crítica percebida influencia positivamente os benefícios percebidos pelos estudantes de contabilidade.

Destaca-se também a parte indireta na qual se encontram a compatibilidade percebida e a complementaridade percebida. A primeira preocupa-se com a compatibilidade que determinado produto poderá oferecer para aumentar sua demanda. Além disso, para que uma rede social (ex.: Instagram) ou um aplicativo de estudos alcance um alto nível de popularidade, precisa-se oferecer um serviço compatível com a vontade de seu usuário para que ele continue a usa-lo e assim ajudar na sua popularização (Chiu et al., 2013).

Para Hsu e Lin (2016) a compatibilidade consiste no usuário perceber que determinado recurso tecnológico irá suprir sua necessidade, ou seja, provocará impactos positivos (benefícios). Assim, percebe-se que a existência de compatibilidade no uso de tecnologias associa-se a intenção de conectividade, pelo usuário de tal meio/ aparato (Abdel-Basset et al., 2018).

A conectividade permeia o nível de aceitação por parte dos estudantes, já que a partir da interação de tarefas de aprendizagem por vias tecnológicas é possível obter benefícios (melhorar suas competências e fomentar o autoaprendizado) (Lim, 2017). A "compatibilidade percebida é o grau em que a IoT é percebida como consistente com valores, necessidades e experiências passadas." (Hsu \& Lin, 2016, p. 519).

Infere-se assim a hipótese $\mathrm{H}_{2 \mathrm{C}}$ : A compatibilidade percebida influencia positivamente os benefícios percebidos pelos estudantes de Contabilidade.

De outra parte, entende-se como complementaridade percebida a forma como determinado produto pode alcançar uma melhor análise dos usuários no momento de escolha para usufruí-lo. Isto é, quais serviços ele irá prestar? O que tornaria produto X melhor que Y na hora da escolha? Ou se o primeiro produto irá oferecer mais serviços complementares do que o segundo? Este tipo de questões foram algo muito comum no final da década de 80 quando os consumidores decidiam qual computador comprar (Hsu \& Lin, 2016).

Segundo Chiu et al. (2013), os recursos tecnológicos propiciam um complemento para que os estudantes possam engajar-se de forma mais interativa com seus colegas e professores.

Postula-se, por tanto, a hipótese $\mathbf{H}_{2 \mathrm{D}}$ : A complementaridade percebida influencia positivamente os benefícios percebidos pelos estudantes de Contabilidade.

As novas tecnologias na educação podem tornar-se uma importante ferramenta para dinamizar o processo de ensino-aprendizagem. Se aplicada de modo responsável e criativo, a tecnologia pode apresentar diferentes benefícios para os alunos e até mesmo para a equipe de educadores. Sendo assim, o uso de equipamentos como softwares e mídias podem contribuir para o desenvolvimento dos alunos e auxiliar os professores no despertar da curiosidade dos estudantes (Silva, Samá \& Lunardi, 2017).

De outra parte, a preocupação com a privacidade das informações faz com que seus usuários usufruam de meios tecnológicos levando-se em consideração que tais dispositivos atuam como promotores de divulgação de informações. Deste modo, contribuem a que haja uma rede de massa usuária na qual podem compartilhar as informações em qualquer momento. Nesse sentido, menciona-se a existência de um impacto quando se aborda a ideia dos benefícios obtidos a partir da preocupação dos estudantes com as informações disponibilizas em rede (Hsu \& Lin, 2016).

Postula-se, então a hipótese H.: A preocupação com recursos interativos na aprendizagem influencia positivamente os benefícios percebidos pelos estudantes de Contabilidade.

A partir das hipóteses construídas na revisão da literatura, elaborou-se o modelo teórico (figura 1) a ser testado nesta investigação. 


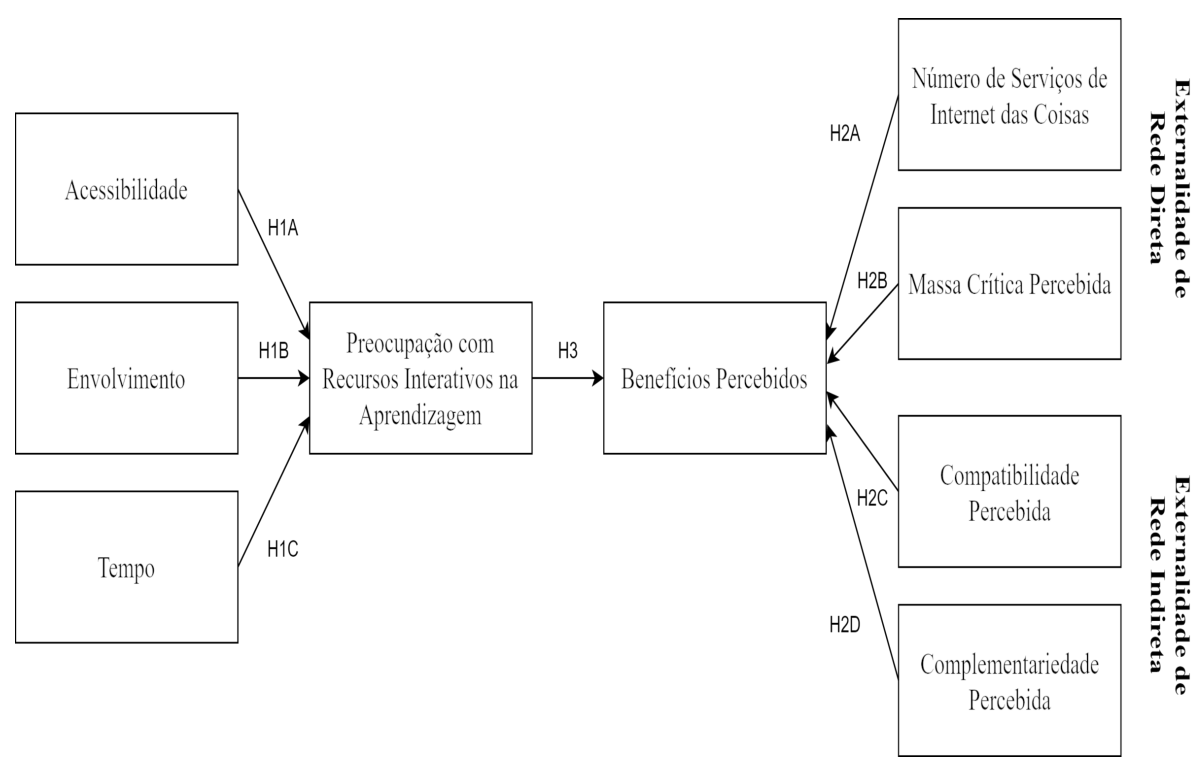

FIGURA 1

Modelo teórico

Fonte: adaptado de Hsu \& Lin (2016) e Watty et al. (2014).

\section{Procedimentos metodológicos}

\section{População e Amostra}

A população $(\mathrm{N})$ compreende 107 discentes, os quais estão regularmente matriculados na disciplina de contabilidade intermediária, situada no segundo semestre da grade curricular do curso de graduação em Ciências Contábeis, de uma IES Federal da região Sul do Brasil. O curso compõe-se por oito semestres, além de configurar-se exclusivamente no período noturno.

A aplicação do questionário in loco ocorreu nos dias 30 de setembro e 01 de outubro de 2019, resultando em uma amostra ( $\mathrm{n}$ ) de 85 respondentes, o que corresponde a $80 \%$ da população. No entanto, destes, em dois questionários houve missings, abrangendo um total de cinco assertivas. Para garantir a preservação da maior quantidade de dados possíveis, observando que os missings correspondem a menos de $5 \%$, tratou-se os dados ausentes inserindo a média dos demais respondentes na mesma assertiva (Nascimento \& Macedo, 2016; Siska, 2018).

No que concerne à idade, a asmotra apresenta um mínimo de 18 e um máximo de 50 anos, com média de 25 anos, moda de 19 anos (10 respondentes) e mediana de 23 anos, o que demonstra que a maior parte da amostra possui idade inferior à média aritmética, isto é, existe a predominância de um público jovem. No que tange ao período letivo, apesar do questionário ter sido aplicado em uma disciplina do segundo semestre, houve discentes que apontaram estar matriculados em mais disciplinas de outro semestre. Este caso aconteceu em aproximadamente $24 \%$ dos casos, nos quais os alunos comentaram estar predominantemente no terceiro, quarto ou quinto semestre.

\section{Instrumento de pesquisa}

Para mensurar os construtos, utilizou-se um questionário estruturado em dois blocos: (i) perfil do respondente, versando a idade e o semestre (apesar de ser em uma disciplina do segundo período, alguns 
discentes estavam cursando mais disciplinas de outro período); e (ii) construtos e suas assertivas. Neste segundo bloco (tabela 1) utilizou-se uma escala likert de seis pontos: (0) não sei responder; (1) discordo totalmente; (2) discordo parcialmente; (3) não discordo nem concordo; (4) concordo parcialmente; e (5) concordo totalmente.

TABELA 1

Construtos e assertivas

\begin{tabular}{|c|c|}
\hline \multicolumn{2}{|r|}{ Número de serviços de IoT } \\
\hline & Eu acho que um bom número de serviços de IoT pode ser frequentemente usado \\
\hline & Eu poderia encontrar facilmente os serviços de IoT \\
\hline & Eu acho que existem muitos pontos de serviços de IoT \\
\hline \multicolumn{2}{|c|}{ Massa crítica percebida } \\
\hline 4 & A maioria das pessoas no meu grupo de colegas usam IoT \\
\hline 5 & A maioria das pessoas na minha comunidade usam serviços de IoT \\
\hline 6 & Minha família/amigos usam regularmente serviços de IoT \\
\hline 7 & A maioria das pessoas que conheço usam serviços de IoT \\
\hline \multicolumn{2}{|c|}{ Compatibilidade percebida } \\
\hline 8 & O uso de IoT é compatível em todos os aspectos do meu estudo \\
\hline С 9 & Usar serviços de IoT se encaixa bem com a maneira que eu gosto de estudar \\
\hline 10 & Serviços de IoT se encaixam no meu estilo de estudo \\
\hline \multicolumn{2}{|r|}{ Complementariedade percebida } \\
\hline 11 & Uma ampla gama de serviços está disponível em IoT \\
\hline 12 & O uso da IoT facilitará vários tipos de trabalhos (por exemplo, pagamentos) \\
\hline \multirow{2}{*}{\multicolumn{2}{|c|}{ Benefícios percebidos }} \\
\hline & \\
\hline 14 & Melhorar meu desempenho nos estudo/vida \\
\hline 15 & Permite que eu realize minhas tarefas da faculdade/vida mais rapidamente \\
\hline 16 & Serviços de IoT são muito úteis para mim \\
\hline \multicolumn{2}{|c|}{ Acessibilidade } \\
\hline 17 & Eu acho que a IoT permite aprender a qualquer hora e lugar \\
\hline 18 & A informação que advém da IoT é tão prontamente disponível que chega fácil até mim \\
\hline 19 & Eu acho fácil que a IoT facilitou mais a aprendizagem \\
\hline \multicolumn{2}{|r|}{ Envolvimento } \\
\hline \multirow{4}{*}{\begin{tabular}{cc} 
& 20 \\
$G \quad 21$ \\
\\
22 \\
\multicolumn{2}{c}{ Tempo } \\
\end{tabular}} & Eu acho que com a IoT meu aprendizado pode evoluir \\
\hline & Eu acho que o uso da IoT pode ser eficaz na minha aprendizagem \\
\hline & Eu acho que o meu envolvimento pode ser o principal desafio para o uso da IoT \\
\hline & \\
\hline 23 & Eu acho que o tempo pode ser meu maior desafio para o uso da IoT \\
\hline H 24 & Eu acho que levaria muito tempo para evoluir meu aprendizado sobre IoT \\
\hline 25 & Eu acho que levaria muito tempo para adotar a IoT \\
\hline \multicolumn{2}{|c|}{ Preocupação com recursos interativos na aprendizagem } \\
\hline \multirow{3}{*}{$\begin{array}{ll} & 26 \\
\text { I } \quad 27 \\
\\
28\end{array}$} & Eu acho que ao usar a IoT vou alcançar melhores resultados na aprendizagem \\
\hline & Eu acho que a IoT afeta positivamente a educação contábil \\
\hline & Eu acho que a IoT na minha aprendizagem pode melhorar meu desempenho \\
\hline
\end{tabular}

Os construtos e respectivos itens de números de serviços (A), massa crítica percebida (B), compatibilidade percebida (C), complementariedade percebida (D) e benefícios percebidos (E) foram adaptados de Hsu e Lin (2016). Por suas vezes, os construtos e indicadores de acessibilidade (F), envolvimento (G), tempo (H) e preocupação com recursos interativos na aprendizagem (I) foram adaptados de Watty et al. (2014).

\section{Técnica de análise dos dados}

A técnica utilizada para a análise dos dados foi a Modelagem de Equações Estruturais, com estimação por Mínimos Quadrados Parciais, mediante o uso do software SmartPLS 3.0. A justificativa para a escolha desta técnica concerne ao tamanho da amostra $(\mathrm{n}<100)$, à ausência da normalidade na distribuição de dados (evidenciada no teste de Kolmogorov-Smirnov (KS), realizado no SPSS 23), bem como à modelagem complexa (diversas variáveis dependentes e independentes) (Bido \& Silva, 2019).

Acerca da representatividade do tamanho da amostra, dois aspectos foram levados em consideração. $\mathrm{O}$ primeiro tange ao critério de 10 vezes a quantidade de caminhos estruturais, ou seja, $10^{*} 8=80$ respondentes (Wong, 2013). O segundo parâmetro consiste na estimação do poder do tamanho, calculado mediante o software $\mathrm{G}^{*}$ Power 3.1.9.4. Pelos pressupostos de Ringle, Silva e Bido (2014) (tamanho do efeito (f.) = 0,15; $\alpha$ de probabilidade de erro $=0,05$; $\operatorname{poder}(1-\beta$ probabilidade de erro $)$ e quatro preditores $)$, a amostra mínima se configura como 85 casos. Desta maneira, a amostra atende aos pressupostos para a rodagem dos dados no SmartPLS.

Referente aos cuidados com o Commom Metod Bias (CMB) realizou-se dois testes para verificar a presença de viés na análise. O primeiro foi o Teste de Fator Único de Harman, no SPSS. Tal teste consiste em uma 
Análise Fatorial Exploratória (AFE) com todos os construtos, no qual um único fator não deve explicar a maior parte de toda variância do modelo. Na rodagem em questão, um único fator representou 34,83\% (Podsakoff, MacKenzie, Lee \& Podsakoff, 2003). O segundo teste foi o de Correlação de Pearson entre os construtos, também no SPPS, no qual nenhum $\mathrm{r}$ deveria ser maior que 0,90 . Foram encontrados valores menores de 0,708, descartando assim a presença de vieses (Pavlou, Liang, Xue, 2007).

\section{Análise dos dados}

\section{Modelo de mensuração}

Na primeira rodagem dos dados, foi observado a composição das cargas fatoriais dos indicadores, no qual índices inferiores a 0.7 foram excluídos para promover o ajuste do modelo (Hair, Hult, Ringle \& Sarstedt, 2016). Nesta perspectiva, excluiu-se as assertivas G22, H23, D11 e D12. No entanto, com a exclusão destas últimas duas, o construto de Complementariedade percebida ficaria com apenas uma variável, sendo assim, optou-se por excluir D13 e consequentemente, o construto.

Partindo do pressuposto da adequação das cargas fatoriais em seus respectivos construtos (todas acima de 0,7 ), prossegue-se para a tabela 2. Neste momento, leva-se em consideração aspectos no contexto da confiabilidade do modelo, como a Composite Reliability (CR), que deve ser superior a 0,7, assim como o Alpha de Cronbach ( $\alpha$ ), que também se espera índices superiores a 0,7. Ambos indicadores foram integralmente atendidos.

TABELA 2

Confiabilidade, validades discriminante e convergente

\begin{tabular}{|c|c|c|c|c|c|c|c|c|c|c|c|c|}
\hline & $\bar{x}$ & CR & $\alpha$ & AVE & $\mathbf{A}$ & B & C & E & F & G & H & I \\
\hline A & 4,16 & 0,840 & 0,727 & 0,637 & 0,798 & & & & & & & \\
\hline B & 3,61 & 0,922 & 0,888 & 0,747 & 0,412 & 0,864 & & & & & & \\
\hline C & 3,74 & 0,923 & 0,873 & 0,800 & 0,429 & 0,334 & 0,894 & & & & & \\
\hline E & 4,12 & 0,911 & 0,854 & 0,774 & 0,424 & 0,373 & 0,632 & 0,880 & & & & \\
\hline F & 4,01 & 0,849 & 0,734 & 0,653 & 0,318 & 0,364 & 0,569 & 0,618 & $\mathbf{0 , 8 0 8}$ & & & \\
\hline G & 4,07 & 0,943 & 0,880 & 0,892 & 0,208 & 0,238 & 0,642 & 0,674 & 0,602 & 0,945 & & \\
\hline H & 2,18 & 0,921 & 0,838 & 0,854 & 0,258 & 0,381 & 0,308 & 0,266 & 0,271 & 0,225 & 0,924 & \\
\hline I & 3,95 & 0,942 & 0,909 & 0,845 & 0,263 & 0,310 & 0,656 & 0,538 & 0,580 & 0,758 & 0,247 & 0,919 \\
\hline
\end{tabular}

Fonte: dados da pesquisa.

A validade convergente, relativa ao construto e sua capacidade de medir de fato o que deveria, foi averiguada por meio da AverageV ariance Extracted (AVE), ou Variância Média Extraída. A AVE deve apresentar valores superiores a 0.5 , o que juntamente com a adequada composição de cargas fatoriais na matriz cruzada (tabela 2), confirma a presença da validade convergente (Fornell \& Larcker, 1981; Hair et al., 2016).

Por sua vez, a validade discriminante, caracterizada pela condição de exclusividade dos indicadores que compõe seus devidos construtos, foi analisada perante o pressuposto das cargas cruzadas (Hair et al., 2016). Neste panorama, foram observados se as cargas cruzadas estavam maiores em seus próprios construtos, bem como se a raiz quadrada da AVE são superiores as correlações das próprias colunas (Fornell \& Larcker, 1981; Hair et al., 2016). 


\section{Modelo estrutural}

Destarte, realizada a adequação e conferência dos pressupostos do modelo, inicia-se a avaliação do modelo estrutural, disposto na tabela 3. Ao que tange às definições do bootstrapping (reamostragens aleatórias para o cálculo da significância), utilizou-se como parâmetro a quantidade de 10.000 interações (Streukens \& LeroiWerelds, 2016).

TABELA 3

Teste de hipóteses

\begin{tabular}{|c|c|c|c|c|c|c|c|c|c|c|}
\hline H & Relação & VIF & $f^{2}$ & $\boldsymbol{\beta}$ & t-statistic & $\mathrm{p}$ value & Aceita-se & $r^{2}$ & $\mathbf{r}^{2} \mathbf{A j}$. & $Q^{2}$ \\
\hline $\mathrm{H} 1 \mathrm{~A}$ & $\mathrm{~F} \rightarrow \mathrm{I}$ & 1,619 & 0,051 & 0,182 & 1,115 & 0,265 & - & \multirow{3}{*}{0,601} & \multirow{3}{*}{0,586} & \multirow{3}{*}{0,331} \\
\hline H1B & $\mathrm{G} \rightarrow \mathrm{I}$ & 1,579 & 0,643 & 0,636 & 4,043 & $0,000^{*}$ & Sim & & & \\
\hline $\mathrm{HlC}$ & $\mathrm{H} \rightarrow \mathrm{I}$ & 1,086 & 0,007 & $-0,055$ & 0,748 & 0,454 & - & & & \\
\hline $\mathrm{H} 2 \mathrm{~A}$ & $\mathrm{~A} \rightarrow \mathrm{E}$ & 1,367 & 0,033 & 0,154 & 1,734 & $0,083^{* *}$ & Sim & \multirow{4}{*}{0,466} & \multirow{4}{*}{0,440} & \multirow{4}{*}{0,447} \\
\hline $\mathrm{H} 2 \mathrm{~B}$ & $\mathrm{~B} \rightarrow \mathrm{E}$ & 1,276 & 0,019 & 0,115 & 1,307 & 0,191 & - & & & \\
\hline $\mathrm{H} 2 \mathrm{C}$ & $\mathrm{C} \rightarrow \mathrm{E}$ & 2,010 & 0,145 & 0,394 & 3,280 & $0,001^{\circ}$ & Sim & & & \\
\hline $\mathrm{H} 3$ & $\mathrm{I} \rightarrow \mathrm{E}$ & 1,792 & 0,043 & 0,203 & 1,797 & $0,072^{* *}$ & Sim & & & \\
\hline
\end{tabular}

Nota. $\mathrm{A}=$ Número de serviços de IoT; $\mathrm{B}=$ Massa crítica percebida $\mathrm{C}=$ Compatibilidade percebida; $\mathrm{D}=$ Complementariedade percebida; $\mathrm{E}=$ Benefícios percebidos; $\mathrm{F}=$ Acessibilidade; $\mathrm{G}=$ Envolvimento; $\mathrm{H}=$ Tempo; I = Preocupação com recursos interativos na aprendizagem; $\mathrm{H}=$ Hipótese; VIF = VarianceInflaction Factor $\mathrm{f}^{2}=$ Tamanho do Efeito; $\beta$ = Coeficiente estrutural; $\mathrm{r}^{2}=$ coeficiente de determinação de Pearson ao quadrado; $\mathrm{r}^{2} \mathrm{Aj}=$ coeficiente de determinação de Pearson ao quadrado ajustado; $Q^{2}=$ valor de StonerGeisser* Aceita-se sig. $<0,01 ; * *$ Aceita-se sig. $<0,10$.

\section{Fonte: dados da pesquisa.}

No contexto da validade preditiva, foram analisados três indicadores: o coeficiente de determinação de Pearson ao quadrado ajustado $\left(r^{2} A j\right.$.); o tamanho do efeito $\left(f^{2}\right)$ e o valor de Stoner-Geisser $\left(Q^{2}\right)$. O R. Aj. Para ambas as variáveis dependentes consegue explicar mais de $40 \%$ do modelo, o que se caracteriza como grande poder de explicação. Por sua vez, o $\mathrm{f}^{2}$ apresenta um forte efeito para $\mathrm{H}_{1 \mathrm{~B}}$, e de pequeno a médio para as demais hipóteses aceitas (Cohen, 1988). Por fim, o Q. deve ser diferente de zero, o que também foi atendido (Bido \& Silva, 2019). Finalmente, analisou-se a possível existência de multicolinearidade (VarianceInflaction Factor - VIF) entre os construtos latentes. Os valores encontrados oscilaram entre 1.086 e 2.010.

\section{Discussão empírica dos dados}

Na figura 2 evidenciam-se as significâncias existentes entre os construtos. Estas estão representadas pelas setas contínua (existência de relação significante) e tracejada (não existência de relação significante).

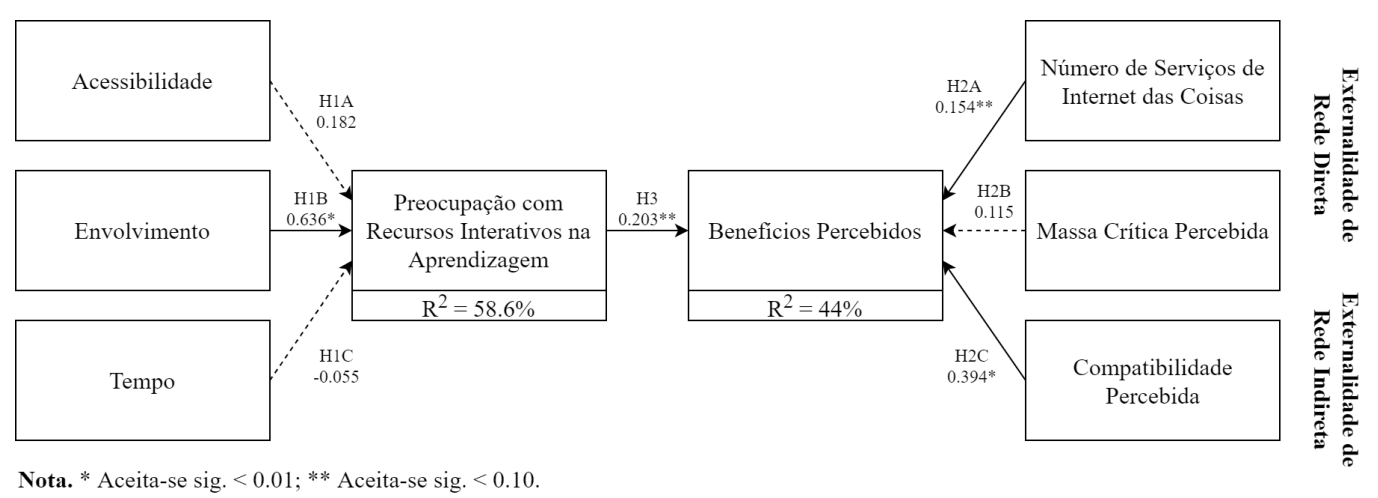

FIGURA 2

Modelo estrutural

Fonte: dados da pesquisa. 
De forma a expor as relações existentes entre os construtos (figura 2) menciona-se que a partir da $\mathbf{H}_{1 \mathrm{~A}}$ não houve relação significativa entre a acessibilidade e a preocupação com recursos interativos na aprendizagem. Isto converge com os argumentos de Kanwal e Rehman (2017), os quais ponderaram que em países com uma tecnologia em crescimento, tal elemento (acessibilidade) torna-se pouco perceptível a influência da tecnologia sobre os estudantes. Para Brennand e Brennand (2012) o Brasil é um dos países que mais tem propiciado a expansão de tecnologias em ambientes educacionais.

Igualmente, a não relação existente entre a acessibilidade e os recursos interativos para o desenvolvimento da aprendizagem não minimiza a relevância dos canais de comunicação no âmbito educacional. Apenas se ratifica que "as novas tecnologias da informação e da comunicação encerram potencialidades positivas ao contribuírem cada vez mais para a integração de todos os cidadãos." (Conforto \& Santarosa, 2002, p. 89).

No que concerne a $\mathrm{H}_{1 \mathrm{~B}}$, infere-se que a mesma foi aceita neste estudo, ou seja, o envolvimento influencia positivamente a preocupação com recursos interativos na aprendizagem. Tal constatação converge com Heiberger e Harper (2008), bem como ratifica os pressupostos de Astin (1984) da pertinência da análise do envolvimento quando o assunto contorna a aprendizagem de estudantes universitários.

A promoção de ambientes de aprendizagem utilizando-se de tecnologias acrescenta as interaçóes entre os estudantes. Estes acabam por desenvolver estruturas comportamentais como, por exemplo, a participação ativa com os colegas de aula, assim como com os professores (Northey, Bucic, Chylinski \& Govind, 2015).

Nesse sentido, infere-se que a inserção de dispositivos móveis para a interação de estudantes é uma forma de tornar o aprendizado mais envolvente. Tal menção converge com Lim (2017) que constatou que a inserção de mecanismos tecnológicos em uma universidade na Malásia, e a imersão e interação dos estudantes como estes meios acarretou impacto positivo na aprendizagem.

Além disso, a proposição de uma influência significativa entre o envolvimento e os recursos interativos ratificam o estudo realizado por Kanwal e Rehman (2017), no qual se encontrou uma tendência de que quando o processo de ensino e aprendizagem é centrado no aluno, ou seja, há possibilidade de que este demonstre maior interesse quando instigado a alguma descoberta e/ou questionamento.

Com relação a $\mathbf{H}_{1 \mathrm{C}}$, não houve uma significância existente entre o tempo e a preocupação com os recursos interativos na aprendizagem. Tal resultado, especificamente, neste estudo condiz com o fato do público em análise ser predominantemente jovem (além de estarem em início do curso), o que faz com que não haja uma dificuldade de adaptação. Dado que, por existir uma facilidade dos jovens com relação ao entendimento de novos meios tecnológicos, este elemento (tempo) não teve um impacto significativo.

Isto condize com Cole, Feild e Harris (2004), que adicionam que acadêmicos em fase final de curso são mais propensos a sentirem o elemento tempo como algo negativo. Isto é, caso o estudante apresente um baixo desempenho ao longo de sua graduação, a realização de tarefas mesmo com a inserção de meios tecnológicos pode não ser capaz de promover uma interação e aprendizagem eficazes. Isto faz com que o impacto da introdução de um recurso interativo não seja percebido.

Ademais, em âmbito contábil tem-se que o tempo é um dos fatores que, segundo os autores são "residuais de uma cultura mais antiga, tornando-se cada vez mais irrelevante" no sentido, de compartilhamento de informações. Refere-se que em virtude de haver uma otimização do tempo para troca de informações, o impacto deste em âmbito acadêmico não se faz expressivo (Watty, Mckay \& Ngo, 2014).

Com referência a $\mathrm{H}_{2 \mathrm{~A}}$ infere-se o aceite da mesma, ou seja, o número de serviços de IoT influencia positivamente os benefícios percebidos. Este achado converge com Katz e Shapiro (1985). Quanto mais recursos forem lançados maiores serão as chances de as pessoas perceberem a existência de benefícios com seu uso. No entanto, diverge dos resultados encontrados por Hsu e Lin (2016), ou seja, no estudo realizado pelos autores não houve uma relação significante entre o número de serviços e os benefícios percebidos.

Além disso, os benefícios percebidos são definidos "como o grau em que uma pessoa acredita que o uso da IoT melhoraria seu desempenho" (Hsu \& Lin, 2016, p. 519). Infere-se assim, que neste estudo, especificamente, houve um grau de concordância maior do que o encontrado na pesquisa realizada por Hsu 
e Lin (2016). Isto é, infere-se que quanto mais recursos tecnológicos forem disponibilizados maiores serão os benefícios percebidos.

Tal achado também converge com Hatane et al. (2019), demonstrando assim uma tendência de que quanto mais ferramentas tecnológicas forem introduzidas no ambiente acadêmico, maiores serão as chances de conectividade entre estudantes e docentes. Deste modo, se propiciam chances de melhorias tanto no que diz respeito ao vínculo entre ambos, bem como uma perspectiva de melhor produtividade dos trabalhos.

No tocante a $\mathbf{H}_{2 \mathrm{~B}}$ OS resultados não suportaram a premissa de que a massa crítica percebida influencia positivamente os benefícios percebidos. Deste resultado pode-se inferir que não há uma percepção de que a maioria dos discentes percebam na mesma intensidade os benefícios promovidos. Estes resultados divergem do encontrado por Hsu e Lin (2016). No entanto, no que tange ao construto compatibilidade percebida $\left(\mathrm{H}_{2} \mathrm{C}\right)$ os achados convergiram com o encontrado pelos referidos autores.

Assim, infere-se que com referência ao construto massa crítica percebida os estudantes não necessariamente precisam estar, em sua maioria, utilizando os recursos tecnológicos para que percebam a existência de benefícios com seu uso. Ao passo que há uma percepção da existência de benefícios no que compreende o construto compatibilidade. Esta se refere ao aceite de que o recurso a ser utilizado supre o que o usuário necessita. Tal ponderação também foi explicitada nos estudos de Chiu et al. (2013) e Hsu e Lin (2016).

A hipótese $\mathbf{H}_{3}$, que refere que a preocupação com recursos interativos na aprendizagem influencia positivamente os benefícios percebidos, foi suportada pelos resultados da pesquisa. Isto é, há um impacto positivo ao inserir recursos tecnológicos em sala de aula, e existe a percepção de que com essa inserção benefícios sejam percebidos. Isto confirma a Silva, Samá e Lunardi (2017). Para Warren et al. (2014) a promoção de recursos interativos entre acadêmicos reflete benefícios em virtude de provocar um engajamento entre os mesmos.

Diante do que fora mencionado pode-se perceber que o impacto mais significativo no tocante ao construto preocupação com recursos interativos na aprendizagem foi com relação ao elemento envolvimento. Exprimese assim que um há uma tendência dos estudantes em atentarem-se quanto ao seu uso devido à interação que esses podem acarretar.

Já com relação ao construto benefícios percebidos os resultados apontaram que os elementos serviços de IoT (intrínseco as externalidades de rede direta) e compatibilidade percebida (intrínseca as externalidades de rede indireta) tiverem um impacto mais expressivo. Neste sentido, no primeiro elemento expressa que quanto mais disponibilidade de ferramentas tecnológicas maiores serão os benefícios para o desenvolvimento da aprendizagem. Já no segundo elemento, este mostra que o aceite de tais ferramentas dependerá do estudante perceber que estas serão indispensáveis para suprir suas necessidades.

\section{Considerações finais}

Este estudo teve como objetivo analisar o efeito da preocupação com a Internet das Coisas na aprendizagem, e seus benefícios percebidos por estudantes do curso de Ciências Contábeis em uma Universidade Pública Federal. Tal preocupação foi corroborada por meio de construtos relacionados à acessibilidade, envolvimento e tempo. Em consonância, os benefícios percebidos sob o viés dos construtos são: números de serviços de IoT; Massa Crítica Percebida; e Compatibilidade Percebida.

As discussões proporcionadas pelos 85 respondentes possibilitaram a visualização dos construtos relacionados à preocupação com os recursos interativos na aprendizagem, sendo eles: acessibilidade; envolvimento e tempo. Houve um impacto mais significativo no construto envolvimento o qual denotou que há a necessidade de uma interação entre os acadêmicos, ou seja, uma participação ativa que busque desenvolver as estruturas comportamentais destes. Deste modo, os estudantes conseguiriam perceber por meio de tal engajamento os benefícios promovidos com a inserção de ferramentas tecnológicos em prol da aprendizagem. 
Neste estudo, ao relacionar os construtos de acessibilidade e tempo com a preocupação com os recursos interativos na aprendizagem, não houve um impacto significativo quanto aos possíveis benefícios percebidos com a inserção de tais recursos. O estudo mostra também que há uma ideia de desenvolvimento, em âmbito nacional, de ambientes educacionais por meio do auxílio da tecnologia.

Em relação ao construto benefícios percebidos, ressalta-se um impacto positivo nos construtos de número de serviços de IoT e da compatibilidade percebida. O primeiro denota que quanto mais recursos tecnológicos forem promovidos na aprendizagem maiores serão os benefícios percebidos pelos discentes. Já o segundo mostra que a aceitação de que os recursos interativos sejam adotados, dependerá da percepção do estudante de que tal ferramenta suprirá sua necessidade.

A investigação contribui ao aproximar empiricamente a teoria da prática, ao fazer corroborações da literatura sobre a IoT em aspectos como o recurso interativo na aprendizagem, ou os benefícios percebidos pelos estudantes de Ciências Contábeis. O estudo acrescenta ainda os antecedentes (externalidades de rede) para a percepção de benefícios na aprendizagem mediante uso de tecnologia, por parte de estudantes de Ciências Contábeis.

No que tange às implicações práticas, os achados podem ser úteis para os discentes compreenderem quais os impactos do uso da IoT nos seus processos de aprendizagem. Ademais, pode contribuir de maneira prática para com o corpo docente, o qual pode buscar novas metodologias que envolvam o uso de IoT dentro da sala de aula.

Uma das limitações do estudo é que não cobriu as percepções de estudantes de instituições privadas, bem como o público que participou da pesquisa. Apenas foram levantados os dados de estudantes pertencentes a uma disciplina (Contabilidade Intermediária). Outra limitação é a ausência de variáveis de controle para o modelo estrutural, tais como a idade, gênero e renda. No entanto, tais limitaçóes podem ser consideradas como sugestões para estudos posteriores.

\section{Considerações éticas}

A pesquisa seguiu procedimentos éticos, em conformidade com o padrão desejado.

\section{Contribuição dos autores}

Todos autores contribuíram de forma igual, desde o planejamento da ideia até a revisão da versão final do manuscrito.

\section{Conflitos de interesse}

Os autores declaram não existir conflitos de interesse.

\section{Agradecimentos}

A pesquisa foi financiada pela Coordenação de Aperfeiçoamento de Pessoal de Nível Superior (CAPES) e pela Fundação de Amparo à Pesquisa do Estado do Rio Grande do Sul (FAPERGS). 


\section{Referências}

Abdel-Basset, M., Manogaran, G., Mohamed, M., \& Rushdy, E. (2018). Internet of things in smart education environment: Supportive framework in the decision-making process. Concurrency and Computation: Practice and Experience, 31(10), 1-12. https://doi.org/10.1002/cpe.4515

Al-Fuqaha, A., Guizani, M., Mohammadi, M., Aledhari, M., \& Ayyash, M. (2015). Internet of things: A survey on enabling technologies, protocols, and applications. IEEE communications surveys \& tutorials, 17(4), 2347-2376. https://doi.org/10.1109/COMST.2015.2444095

Astin, A. W. (1984). Student involvement: A developmental theory for higher education. Journal of college student personnel, 25(4), 297-308. https://psycnet.apa.org/record/1985-18630-001

Bido, D. de S., \& Silva, D. da. (2019). SmartPLS 3: especificação, estimação, avaliação e relato. Administração: Ensino e Pesquisa, 20(2), 1-31. https://doi.org/10.13058/raep.2019.v20n2.1545

Brennand, E. G., \& Brennand, E. de G. (2012). Inovações tecnológicas e a expansão do ensino superior no Brasil. Revista Lusófona de Educação, (21), 179-198. https://revistas.ulusofona.pt/index.php/rleducacao/article/view/3087

Chiu, L. B. F., Ramírez, N. I. H., \& Ruiz, M. V. (2013). Facebook: Una experiencia universitaria. PAAKAT: Revista de Tecnologia y Sociedad, 3(5), 1-7. http://www.udgvirtual.udg.mx/paakat/index.php/paakat/article/view/205 1292

Cohen, J. (1988). Statistical Power Analysis for the Behavioral Sciences, 2. ed. New York: Psychology Press.

Cole, M. S., Feild, H. S., \& Harris, S. G. (2004). Student learning motivation and psychological hardiness: Interactive effects on students' reactions to a management class. Academy of Management Learning \& Education, 3(1), 64-85. https://doi.org/10.5465/amle.2004.12436819

Conforto, D., \& Santarosa, L. M. C. (2002). Acessibilidade à Web: Internet para todos. Informática na educação: teoria ¿ prática, 5(2), 87-102. https://lume.ufrgs.br/handle/10183/20987

Cornel, C-E. (2015). The role of Internet of Things for a continuous improvement in education. Hyperion Economic Journal, 2(3), 24-31. http://hej.hyperion.ro/articles/HEJ\%20nr2(3)_2015.pdf\#page=25

David, O. N., Helou, A. M., \& Rahim, N. Z. A. B. (2012). Model of perceived influence of academic performance using social networking. International Journal of Computers \& Technology, 2(2), 24-29. https://doi.org/10.24 297/ijct.v2i1.2612

Fornell, C. \& Larcker, D. (1981). Evaluating structural equation models with unobservable variables and measurement error. Journal of Marketing Research, 18(1), 39-50. https://doi.org/10.2307/3151312

Ghani, E. K., \& Muhammad, K. (2019). Industry 4.0: Employers' Expectations of Accounting Graduates and Its Implications on Teaching and Learning Practices. International Journal of Education and Practice, 7(1), 19-29. https://doi.org/10.18488/journal.61.2019.71.19.29

Gupta, S., \& Mela, C. F. (2008). What is a free customer worth? Armchair calculations of nonpaying customers' value can lead to flawed strategies. Harvard Business Review, 86(11), 102-138. https://pubmed.ncbi.nlm.nih.gov/19 009724/

Hair Jr., J. F., Hult, G. T. M., Ringle, C. M., \& Sarstedt, M. (2016). A Primer on Partial Least Squares Structural Equation Modeling (PLS-SEM), $2^{\text {nd }}$ ed, SAGE Publications.

Hatane, S. E., Johari, I. V. D., Valencia, J., \& Prayug, L. E. (2019). The acceptance of accounting students on the use of Internet of Things. Advances in Economics, Business and Management Research, 103, 273-278. https://doi.o $\mathrm{rg} / 10.2991 /$ teams-19.2019.44

Heiberger, G., \& Harper, R. (2008). Have you Facebooked Astin lately? Using technology to increase student involvement. New directions for student services, (124), 19-35. https://doi.org/10.1002/ss.293

Hsu, C.-L., \& Lin, J. C.-C. (2016). An empirical examination of consumer adoption of Internet of Things services: Network externalities and concern for information privacy perspectives. Computers in Human Behavior, 62, 516-527. https://doi.org/10.1016/j.chb.2016.04.023 
Kanwal, F., \& Rehman, M. (2017). Factors affecting e-learning adoption in developing countries-empirical evidence from Pakistan's higher education sector. IEEE Access, 5, https://doi.org/10968-10978

Katz, M. L., \& Shapiro, C. (1985). Network externalities, competition, and compatibility. The American economic review, 75(3), 424-440. https://www.jstor.org/stable/1814809

Lim, W. N. (2017, April). Improving student engagement in higher education through mobile-based interactive teaching model using socrative. In 2017 IEEE Global Engineering Education Conference (EDUCON) (pp. 404-412). IEEE. https://ieeexplore.ieee.org/document/7942879

Lin, K. Y., \& Lu, H. P. (2011). Why people use social networking sites: an empirical study integrating network externalities and motivation theory. Computers in Human Behavior, 27(3), 1152-1161. https://doi.org/10.101 6/j.chb.2010.12.009

Lin, C., \& Bhattacherjee, A. (2008). A. Elucidating individual intention to use interactive information technologies: The role of network externalities. International Journal of Electronic Commerce, 13(1), 85-108. https://doi.org /10.2753/JEC1086-4415130103

Lou, H., Luo, W., \& Strong, D. (2000). Perceived critical mass effect on groupware acceptance: a revised technology acceptance model. European Journal of Information Systems, 9(2), 91-103. https://doi.org/10.1057/palgrave.e jis. 3000358

Nascimento, J. C. H. B. do., \& Macedo, M. A. da S. (2016). Modelagem de Equações Estruturais com Mínimos Quadrados Parciais: um Exemplo da Aplicaçãodo SmartPLS ${ }^{\circ}$ em Pesquisas em Contabilidade. Revista de Educação e Pesquisa em Contabilidade, 10(3), 289-313. https://doi.org/10.17524/repec.v10i3.1376

Northey, G., Bucic, T., Chylinski, M., \& Govind, R. (2015). Increasing student engagement using asynchronous learning. Journal of Marketing Education, 37(3), 171-180. https://doi.org/10.1177/0273475315589814

Pavlou, P., Liang, H., \& Xue, Y. (2007). Understanding and mitigating uncertainty in online exchange relationships: A principal-agent perspective. MIS Quarterly, 31(1), 105-136. https://doi.org/10.2307/25148783

Peleias, I. R., Guimarães, E. R., Chan, B. L., \& Carlotto, M. S. (2017). A síndrome de Burnout em estudantes de ciências contábeis de IES Privadas: pesquisa na cidade de São Paulo. Revista de Educação e Pesquisa em Contabilidade, 11(1), 30-51. https://doi.org/10.17524/repec.v11i1.1468

Podsakoff, P. M., Mackenzie, S. B., Lee, J. Y., \& Podsakoff, N. P. (2003). Common method biases in behavioral research: A critical review of the literature and recommended remedies. Journal of applied psychology, 88(5), 879-903. ht tps://doi.org/10.1037/0021-9010.88.5.879

Ringle, C. M., Silva, D. da., \& Bido, D. (2014). Modelagem de Equações Estruturais com Utilização do Smartpls. Revista Brasileira de Marketing, 13(2), 56-73. https://doi.org/10.5585/remark.v13i2.2717

Schwab, D., \& Freitas, C. C. G. (2016). Tecnologia social: implicações e desafios da implantação. Revista Tecnologia e Sociedade, 12(26), 42-60. https://doi.org/10.3895/rts.v12n26.3794

Silva, B. H. P., Samá, S., \& Lunardi, G. L. (2017). Motivos de uso e Benefícios Percebidos pelos Estudantes do Ensino Superior no uso dos Dispositivos Móveis no Ambiente Educacional. RENOTE-Revista Novas Tecnologias na Educação, 15(2), 1-10. https://doi.org/10.22456/1679-1916.79267

Silva, S. de C. R., \& Szesz Junior, A. (2018). Internet das coisas na educação: uma visão geral. Ensino de Ciências e Tecnologia em Revista, 2(1), 57-69. https://dx.doi.org/10.31512/encitec.v8i2.2717

Siska, L. (2018). How Strategic Priorities Are Reflected in Features of Strategic Performance Measurement System? Engineering Economics, 29(5), 591-600. https://doi.org/10.5755/j01.ee.29.5.17463

Streukens, S., \& Leroi-Werelds, S. (2016). Bootstrapping and PLS-SEM: A step-by-step guide to get more out of your bootstrap results. European Management Journal, 34(6), 618-632. https://doi.org/10.1016/j.emj.2016.06.003

Tachizawa, T., \& Andrade, R. O. B. (2003). Tecnologias da informação aplicadas às instituiçôes de ensino e às universidades corporativas. São Paulo: Atlas.

Warren, A. M., Sulaiman, A., \& Jaafar, N. I. (2014). Social media effects on fostering online civic engagement and building citizen trust and trust in institutions. Government Information Quarterly, 31(2), 291-301. https://doi .org/10.1016/j.giq.2013.11.007 
Watty, K., Mckay, J., \& Ngo, L. (2014). Innovative teaching, learning and assessment in accounting education: Engaging with digital technologies that enhance student learning. Deakin University, Australia, Melbourne. https://www .deakin.edu.au/_data/assets/pdf_file/0007/296413/cpareport.pdf

Wong, K. K. K. (2013). Partial least squares structural equation modeling (PLS-SEM) techniques using SmartPLS. Marketing Bulletin, 24(1), 1-32. http://marketing-bulletin.massey.ac.nz/v24/mb_v24_t1_wong.pdf

Wu, J., Xiong, F., \& Li, C. (2019). Application of Internet of Things and blockchain technologies to improve accounting information Quality. IEEE Access, 7, 100090-100098. https://ieeexplore.ieee.org/document/8769831

\section{Notas}

* $\quad$ Artigo de pesquisa científica e tecnológica.

Licencia Creative Commons CC BY 4.0

Para citar este artigo: da Matta AJG, Fernandes CMG, Frare AB, Quintana AC. Internet das Coisas como recurso interativo na aprendizagem: Benefícios percebidos por discentes de Ciências Contábeis. Cuadernos de Contabilidad, 22. https://doi.org/10.11144/Javeriana.cc22.icri 